\title{
Las primeras profesionales del arte peruano en los escritos de Elvira García y García
}

\author{
The early professionals in peruvian art in the writings of Elvira \\ García y García
}

\author{
Sofía Karina Pachas Maceda \\ Universidad Nacional Mayor de San Marcos, Lima, Perú \\ Contacto: spachasm_ac@unmsm.edu.pe \\ https://orcid.org/0000-0001-8736-8534
}

\section{Resumen}

En 1924, Elvira García y García publicó un libro fundamental en la historiografía de las mujeres en el Perú titulado: La mujer peruana a través de los siglos: serie historiada de estudios y observaciones. Se trata de una ambiciosa selección de más de 300 biografías distribuidas en dos tomos.

Este artículo rescata algunas de esas biografías, enriqueciéndolas con notas periodísticas que García y García publicó en reconocidos medios de prensa; escritos que son hoy una radiografía del momento que vivieron las primeras profesionales de las artes plásticas en el Perú.

Palabras claves: Biografías; Artistas peruanas; Siglo XIX; Siglo XX; Crítica de artel

\begin{abstract}
In 1924, Elvira García y García published a book which was meant to be essential in the history of women in Peru: The Peruvian Woman through the centuries historical reviews of studies and comments, an astonishingly detailed and accurate selection of more than 300 biographies compiled in two volumes.

This article focuses on some of these biographies, enriched with reviews that García y García published in well-known and reputed newspapers. Such biographies represent a reflection of the times lived by the first professionals of fine arts in Peru.
\end{abstract}

Keywords: Biographies; Peruvian artists; XIX century; XX century; Critic.

Recibido: 19.05.19

Aceptado: 07.11.19 


\section{Introducción}

Oscuridad, olvido y silencio son algunas de las palabras que suelen asociarse a los estudios relacionados con el quehacer de la mujer a lo largo de la historia. Si esto es todavía hoy una realidad no es difícil imaginar cómo era la situación en la segunda década del siglo pasado. Así lo expresó Elvira García y García en las primeras páginas de La mujer peruana a través de los siglos serie historiada de estudios y observaciones en las que añadió que era un acto de estricta justicia conocer lo que la patria le debe a la mujer (García y García, 1924a).

Elvira García y García (Lambayeque, 1862-Lima, 1951) no era una desconocida cuando publicó esta obra. Hija del matrimonio entre el contraalmirante Aurelio García y García y Eulogia Best, su formación educativa la inició en su tierra natal y la continuó en Lima, dedicándose a partir de los 18 años a la docencia. Seguidora de las teorías educativas de Federico Froebel, uno de sus principales aportes en este campo fue la apertura, en 1902, del primer kindergarten en el Perú.

De manera paralela a su carrera de educadora, desde fines del siglo XIX publicó libros ${ }^{1}$ y ya en el siglo XX colaboró en el diario El Comercio y en la revista Mundial. Así, en ambas actividades destacó su consagración a la mujer; tanto en la formación de las niñas como en sus escritos demuestra su compromiso con la labor femenina en todos los ámbitos. Desde la prensa, las alienta a mejorar y en más de una oportunidad hace un llamado a las autoridades y a particulares para colaborar en el desarrollo social de las peruanas ${ }^{2}$.

Al contrastar los estudios en los que ha sido mencionada Elvira García y García es notorio que su destacado quehacer como educadora ${ }^{3}$ ha opacado su faceta de cronista cultural que aún no ha sido explorada en su verdadera magnitud. Al respecto, la investigadora Aída Balta, en Presencia de la mujer en el periodismo escrito peruano (1821-1960), la integra entre las asiduas colaboradoras en periódicos y revistas de las primeras décadas del siglo XX y destaca su propuesta a "que la mujer se incorpore al campo laboral y cultural del país" (Balta, 1998, p. 134). 
Por ello, el objetivo de este artículo es poner en valor los escritos de Elvira García y García, específicamente los dedicados al arte peruano ejecutado por mujeres. Si bien el eje es el capítulo "La mujer en las bellas artes" incluido en el libro La mujer peruana a través de los siglos ${ }^{4}$, esta información es complementada con sus artículos periodísticos, pues algunos de ellos fueron la base sobre la cual se escribió la biografía de las artistas. Es necesario destacar que los escritos seleccionados fueron dados a conocer durante la década de 1920, época relevante para el arte peruano dado que por esos años existió un interés especial por cubrir las actividades realizadas en la recién inaugurada Escuela de Bellas Artes del Perú (1918).

De esta forma, los artículos de García y García se insertan en un medio cultural en el que otros autores también incursionaban en la crítica de arte peruano. Sin embargo, la autora se va a distinguir de ellos por su particular estilo en el que, incluso, deja de lado las salas de exhibición e ingresa en el mundo íntimo del taller de la artista. A partir de ello, planteamos la principal pregunta que guiará este estudio: ¿de qué manera contribuyen los escritos de Elvira García y García a conocer el quehacer de las primeras profesionales del arte peruano?

El asunto plasmado en este artículo está vinculado a las investigaciones relacionadas con el arte y género, tema que empezó a cobrar interés en la historia del arte desde el artículo pionero de Linda Nochlin “¿Por qué no han existido grandes artistas mujeres?", publicado originalmente en 1971, y cuya propuesta renovadora fue la de cuestionar lo aparentemente "natural" en la historia del arte. Es decir, que la actividad artística fue un campo exclusivamente masculino dado que "las mujeres son incapaces de llegar a la grandeza" (Nochlin, 2007, p. 18) y pone en evidencia que las razones de esa supuesta superioridad masculina no se relacionaban con un don especial, sino con la falta de estímulo que recibían las mujeres en el ámbito privado (familia) y al casi nulo compromiso público (instituciones educativas, museos, etc). Situación social de exclusión que fue cambiando a medida que avanzaba el siglo XX, aunque no podemos asegurar se haya superado por completo en el campo artístico. 
En tal sentido, los escritos de Elvira García y García permiten no solo visualizar a un grupo de mujeres dedicadas al arte en un contexto de cambio, entre las aficionadas y las profesionales del arte peruano, sino también dan a conocer esos obstáculos y alicientes que permitieron que algunas mujeres desarrollasen su actividad como estudiantes y luego como artistas profesionales ${ }^{5}$.

\section{La mujer en la historia peruana}

En 1909, Elvira García y García fundó la revista El Hogar y la Escuela en cuyas páginas incluyó la sección “Galería de mujeres célebres”. Desde allí empezó a redactar breves biografías de peruanas, las cuales serán el germen de las páginas de La mujer peruana a través de los siglos.

La redacción de estas primeras biografías las compiló, corrigió y aumentó años después cuando publicó su libro en dos tomos. El contexto en el que vio la luz fue en el quinto año de los once que permaneció en el poder Augusto B. Leguía (1919-1930). Caracterizado en lo económico por la estrecha relación con Estados Unidos, uno de los propósitos de la entonces llamada Patria Nueva era la "ruptura del control político de las élites civilistas", para incorporar a las clases trabajadoras e indígenas (Contreras \& Cueto, 2013, p. 244).

En tanto, en lo relacionado con la historia de las peruanas, es interesante constatar que desde el primer año del gobierno de Leguía empezaron a tener protagonismo en el reclamo social, cuando en 1919 se realizó un gran mitin de mujeres en el que se destacó la necesidad de una lucha conjunta en beneficio de las demandas obreras (Guardia, 2002). Años antes, 1914, María Jesús Alvarado fundó Evolución Femenina, el primer colectivo feminista en el Perú y una década después hacía lo propio la literata Zoila Aurora Cáceres con Feminismo Peruano, este último de importancia decisiva en la primera mitad de 1930, cuando logra introducir en el debate de la Asamblea Constituyente el voto de la mujer (Cáceres, 1909).

Así, García y García eligió un momento de especial coyuntura para dar a conocer su libro, pues en 1924 Lima fue la sede de la Segunda Conferencia Panamericana de Mujeres y fue este el espacio propicio para presentar La mujer 
peruana a través de los siglos. Celebrado entre el 21 de diciembre de 1924 y el 4 de enero de 1925, este simposio congregó a representantes de doce países, quienes intercambiaron experiencias sobre el estado jurídico de la mujer y plantearon alternativas para lograr la igualdad frente a la ley.

Con este marco de reivindicación de la mujer, Emilio Gutiérrez de Quintanilla fue el elegido para presentar el texto. Destacado intelectual a quien la trayectoria de la escritora no le era indiferente, así lo señaló:

La señorita García y García trae a su libro sentimientos que formó en sus alumnas, durante largos años de magisterio, para infiltrarlos en la vida pública, fuera de la enseñanza reglamentaria y privada. En él completa la doctrina moral y social que la civilización profesa, con el glorificado ejemplo de numerosas damas que, culminando en saber y virtudes cívicas, trajeron al proceso histórico de nuestra nacionalidad lo más valioso de la cooperación femenina, desde la influencia literaria y artística ejercida en múltiples aspectos, hasta la acción heroica y memorable del voluntario sacrificio. (Gutiérrez de Quintanilla, 1924, p. 10)

Además de destacar los valores atribuidos a las mujeres, lo interesante de este comentario es que García y García educó también en identidad, en este caso al difundir entre sus alumnas lo logrado por otras mujeres, contribuyendo así a una conciencia de género. Esto confirma lo renovadora que era la propuesta educativa planteada por nuestra protagonista, ya que en la actualidad se analiza la manera cómo revertir el currículo androcéntrico a partir de reconocer las contribuciones sociales y la experiencia cultural de las mujeres (Colás Bravo \& Jiménez Cortés, 2006). Compromiso asumido por García y García desde inicios del siglo XX.

Por otro lado, no es casual que el autor resalte la incursión femenina en las expresiones artísticas, pues entre los escritos de Gutiérrez de Quintanilla sobresalen artículos de crítica de arte, en los que en más de una oportunidad comentó obras ejecutadas por manos de mujeres. Inclusive entre sus numerosas publicaciones se encuentra Meditaciones sobre la amada costilla, texto en el que expresó su adhesión al movimiento feminista en el Perú. 
El máximo aporte del libro de García y García es el de poner en relieve, con nombre y apellido, a las mujeres que contribuyeron a forjar el Perú. No está de más señalar que entre los diccionarios biográficos publicados en el país antes de 1924, apenas son consignados algunos nombres femeninos; estos se limitan, en algunos casos como en el voluminoso Diccionario histórico biográfico del Perú de Manuel de Mendiburu ${ }^{6}$, a las biografías de religiosas y, con ello, relegan a la mujer a un rol pasivo, sin ninguna aparente relevancia en la actividad civil y pública.

Sin embargo, no todos comprendieron, ni en su momento ni con posterioridad, el significado de una propuesta como la de La mujer peruana a través de los siglos. Así lo demuestra el historiador Raúl Porras Barrenechea en Fuentes históricas peruanas, cuando minimizó la obra calificándola como "un arsenal de ingenuidades románticas indocumentadas” (Porras Barrenechea, 1954, p. 326).

Pero iniciemos el análisis del libro. Se trata de un compendio biográfico desde el Antiguo Perú hasta las primeras dos décadas del siglo XX. Si bien es cierto que los datos relacionados con las mujeres en épocas remotas son casi esbozos en los que la falta de fuentes es notoria, consideramos que es una licencia que la autora se atribuye en su afán de mostrar una línea de tiempo ininterrumpida en la que las peruanas han tenido distintos protagonismos. Sin embargo, conforme avanza en las etapas históricas, los datos de las protagonistas se vuelven más exactos, con la rigurosidad que merecen. Así, cuando escribe sobre Inés Muñoz de Rivera, viuda del conquistador Antonio de Rivera, consignó información sobre su actividad filantrópica en la que destacó la fundación del monasterio de la Concepción, parte de cuyo local todavía hoy puede apreciarse en Lima.

Por ello, las últimas páginas del segundo tomo son de especial interés para conocer el movimiento social femenino en Lima de la segunda década del siglo $\mathrm{XX}$, pues en ellas la autora brinda abundantes datos sobre la actividad de diversos colectivos, desde los específicamente creados para las mujeres trabajadoras hasta los de proyección artístico-cultural. Por ejemplo, uno de los que consigna es el Consejo Nacional de Mujeres del Perú, agrupación que reunía a 35 asociaciones cuyos propósitos coincidían en la necesidad de capacitar moral, intelectual y económicamente a la mujer. Ella misma era miembro de este Consejo. 
Por todo ello, el libro de García y García puede considerarse una genealogía del accionar de la mujer en el Perú y como proceso de organización de este tipo es posible ligarlo a una idea de pertenencia, de identidad. Una identidad de dos tipos: de género y nación.

Sobre esta iniciativa es interesante relacionarla con la labor de difusión que habían empezado a hacer las ya mencionadas Zoila Aurora Cáceres y María Jesús Alvarado; en el caso de Cáceres daba a conocer, desde principios del siglo $\mathrm{XX}$, la labor de mujeres en diversos contextos y lugares, las mismas que fueron protagonistas de su primer libro Mujeres de ayer y hoy (Cáceres, 1909). Mientras que Alvarado hizo lo propio con peruanas destacadas a las que en su Manifiesto de 1911 denominó "campeonas del movimiento feminista intelectual en el Perú"; precisamente Elvira García y García era una de ellas (Alvarado Rivera, 2011, p. 51).

Una investigadora que ha reflexionado sobre este tema es Rocío del Águila, quien aborda la idea de la genealogía entre intelectuales y hace notar los actos simbólicos de parentesco (bautizos literarios y dedicatorias en publicaciones) presentes en las relaciones interpersonales de las escritoras en el Perú de fines del siglo XIX. El estudio concluye que estas intromisiones en la dinámica de poder beneficiaron a sus "descendientes culturales", quienes emprenden sus propuestas literarias partiendo de estas bases (Águila, 2013). En el caso de La mujer peruana a través de los siglos, se trata de una genealogía de peruanas sin discriminar en oficio, ni aporte, por más mínimo que pareciera; aquí el lazo de filiación es el género y el acto de parentesco, un accionar.

Punto crucial en la propuesta educativa y de difusión emprendida por Elvira García y García es una renovación del modelo de mujer, de la tradicional identidad femenina. Entendida la identidad de género como la noción que uno tiene de su propia masculinidad o feminidad y que "hace referencia a atributos determinados cultural e institucionalmente, como intereses predominantes, maneras, sensibilidad emocional, agresividad" (Person, 1999, p. 67). Sin embargo, tanto el hombre como la mujer pueden salirse del guion esperado y desafiar esos atributos convencionales; así, la autora a partir de algunas biografías promueve 
una renovación del estereotipo clásico de la vida de las mujeres, cambio en el que la educación tenía un rol relevante. Así lo expresó en la conferencia "Tendencias de la educación femenina" de 1908, en la que las anima a continuar su aprendizaje luego de concluir estudios escolares:

La mujer debe y puede seguir todas las carreras liberales, eligiendo la que esté más en armonía con sus aspiraciones, con su vocación y con sus recursos. Las puertas de la Universidad le están hoy del todo franqueadas y pueden consagrarse con libertad a estudiar Literatura, Ciencias Físicas y Naturales, Matemáticas, Derecho, Medicina, Farmacia, Industrias, Comercio, Bellas Artes, Pedagogía, Filosofía, etc., etc. [...] Déjesele á cada cual, hombre ó mujer, la responsabilidad de seguir la carrera que más le convenga, ó la que responda mejor á sus aspiraciones. (García y García, 2013, p. 239)

Es importante señalar que la propuesta de García y García tenía un propósito claro: mejorar la formación educativa de las mujeres dado que ellas luego serían las encargadas de la educación de sus hijos. Es decir, planteaba un cambio pero con un propósito maternal. Asimismo, es necesario especificar que la autora se distanció de las propuestas feministas y "las rechazó explícitamente", pues estaba en contra del enfrentamiento contra los varones (Mannarelli, 2013, p. 51).

Pero volvamos a la identidad y su relación con la nación en el libro de García y García. Este punto toma mayor fuerza si tenemos en cuenta que fue publicado en 1924, año en que se conmemoraba el Centenario de la Batalla de Ayacucho y a tres de haber hecho lo propio con la Independencia del Perú. Momento aprovechado por la autora para poner en evidencia, tanto entre sus congéneres como entre los hombres, el aporte de las peruanas en la historia del Perú.

\section{La mujer en las Bellas Artes}

Por todo ello, La mujer peruana a través de los siglos fue un libro singular para la época y, para el caso específico de la historia del arte peruano, su aporte resulta aún más significativo, pues hasta ese momento ningún diccionario biográfico en el Perú había consignado el nombre de una artista plástica. Uno de estos libros es el de Juan Pedro Paz Soldán, quien en Diccionario biográfico de peruanos contemporáneos (1917) insertó la biografía de nueve artistas. Cuatro años después 
sacó a la luz la segunda edición en la que colocó la vida de once artistas plásticos, ninguna de las dos ediciones considera a una artista.

A pesar de que García y García se consideraba una "profana” en materia artística, las biografías y sus artículos publicados sobre artes plásticas demuestran lo contrario. En esos escritos no es difícil percatarse de sus conocimientos y de una cultivada sensibilidad por las expresiones artísticas.

¿Quiénes o qué contribuyó a este gusto por el arte? Las posibles influencias recibidas por García y García nos llevan a indagar en su círculo de amistades, siendo la compartida con Elena Ortiz de Zevallos (figura 1) la más relevante. Miembro de una acaudalada familia, propietaria de la más importante colección de arte europeo de la época, Elena era también pintora. En la década de 1920, la pinacoteca Ortiz de Zevallos podía ser admirada en una improvisada sala de exhibición en la casa familiar, habilitada por la misma Elena, quien además era la conservadora y restauradora de los lienzos (García y García, 1926, p. 1). Sin duda, sus conocimientos sobre historia y arte fueron una ayuda decisiva para la escritora, quien no vacila en expresarlo en la dedicatoria de La mujer peruana a través de los siglos: "A la buena amiga, con cuya exquisita y delicada conversación he podido adquirir buen número de referencias, las que, sin ese auxilio, habrían quedado sepultadas, en las tinieblas del olvido. Con todo mi cariño y agradecimiento le ofrezco este libro" (García y García, 1924a).

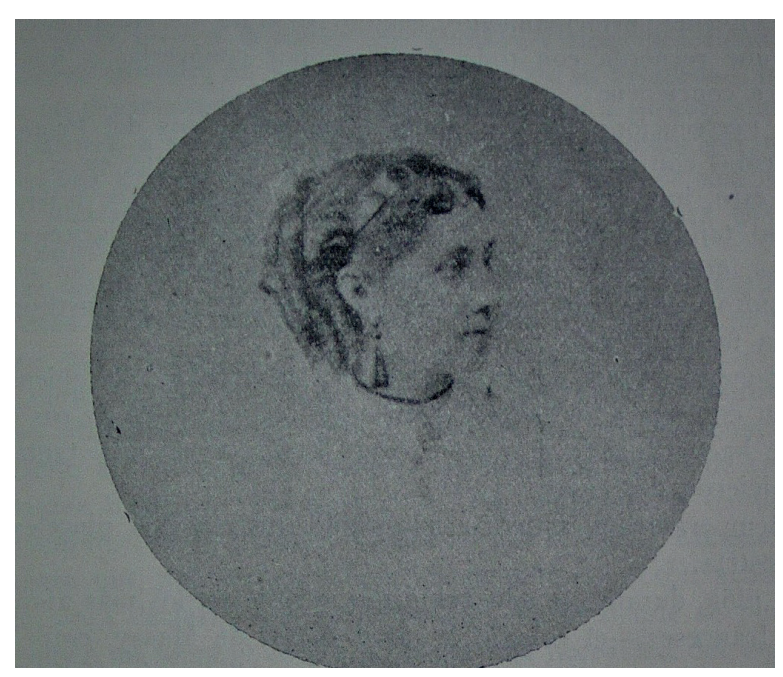

Figura 1. Elena Ortiz de Zevallos y Tagle 
La solidez de esta amistad cultivada a partir de la afinidad por el arte no resulta casual dado que Elvira García y García consideró esta expresión humana de vital importancia en el desarrollo, y en el caso de la enseñanza femenina era de mayor interés. Así lo escribe en su libro Actividad femenina, en el cual señaló que las bellas artes deberían ocupar un lugar preferente, pues a través del sentido estético "puede formarles el hábito de las ideas moderadas y superiores; de la elevación de los sentimientos desinteresados" (García y García, 1928, p. 13).

Dada esta importancia que le otorga, no sorprende que el cuarto acápite del segundo tomo esté dedicado a la mujer en las bellas artes. Así, este apartado se compone de 65 páginas dedicadas a 21 biografías de 18 pintoras, una escultora y una litógrafa ${ }^{7}$. El orden en que aparecen no responde al alfabético, sino al generacional; así, la lista está encabezada por Rebeca Oquendo, nacida en 1847 y la pintora más conocida del Perú decimonónico ${ }^{8}$. Respecto a los datos consignados en las vidas de las biografiadas habría que hacer la salvedad de que García y García omite las fechas de nacimiento; en tal sentido, es solo a partir de la lectura y la ocasional cita de algún año o evento que se deduce la época en que la protagonista inicia su actividad.

Antecede a las biografías una sucinta introducción en la que la autora contextualiza el escenario en el cual las limeñas habían recibido el aprendizaje artístico. En esas líneas se refirió a las lecciones de dibujo en los colegios y las esporádicas clases con profesores particulares, oferta educativa que fue incrementándose a medida que avanzó el siglo XX, hasta llegar a 1919. Ese año, el Estado abrió las puertas de la Escuela Nacional de Bellas Artes, a la que denominó como "santuario", lugar en donde las alumnas reciben "no solo la dirección artística teórica y práctica, sino lo que es más importante, la ilustración científica y superior de las bellas artes" (García y García, 1924a, p. 512).

El énfasis en la Escuela de Bellas Artes no era exagerado. El Perú fue uno de los últimos países en la región en fundar su centro de enseñanza artística oficial. Por ello, los primeros años de funcionamiento, tiempo en que sale a luz este libro, fue una época en la cual la prensa cubría con expectativa lo realizado por los estudiantes, 
primeras generaciones de artistas formados en suelo peruano. En este contexto tiene sentido la importancia que la autora otorga a las alumnas de la Escuela, pues de las 21 biografías, doce eran de egresadas o todavía alumnas de ese centro.

Entre ellas son muy pocas las que han logrado trascender la barrera del tiempo. La historia del arte peruano ha dedicado escasas páginas a las artistas de este período, época de tránsito entre la práctica amateur y la profesional de las artes. De esta forma, son tres las que hasta el momento han sido relativamente más estudiadas, ellas son: Julia Codesido, Elena Izcue y Rebeca Oquendo?.

Algunas de las biografías incluidas en el libro tienen su origen en artículos publicados a propósito de la visita a exposiciones y, por ello, al ser comentario del momento, sus escritos son crítica de arte. Probablemente, su oficio de pedagoga influyó a que se oriente por un género literario cuyo origen es más didáctico, pues cumplía con el objetivo de "acercar" la obra a un público no especializado.

En las siguientes líneas desarrollamos las principales características de las biografías escritas por Elvira García y García, las cuales permiten definir su estilo de escritura biográfica y, además, formarse un panorama del ambiente cultural que vivieron las primeras profesionales del arte pictórico en el Perú.

\section{La tinta y el pigmento}

A lo largo de la historia, la tinta y el pigmento han sido y continúan siendo los insumos mediante los cuales escritores y pintores han materializado amores, miedos y sus particulares visiones del mundo.

Estos procesos creativos independientes, que se han entrecruzado desde la Antigüedad, tienen un momento de auge en Francia del siglo XIX, cuando los concurridos salones generan gran expectativa, lo que repercute en la génesis de un mercado del arte nunca antes visto. En dicho momento, al que algunos investigadores llaman "democratización del arte", los críticos tienen un rol decisivo, pues son los llamados a fomentar la compraventa del arte, responsables del éxito y fracaso de los artistas y de contribuir, quiéranlo o no, a formar el gusto estético en la sociedad. 
El escenario cultural del Perú de inicios del siglo XX, al igual que otros países latinoamericanos, estaba muy lejos de parecerse a la actividad parisina. El ambiente artístico era mínimo, pocas salas, escasos compradores y escuetos comentarios en prensa. Sin embargo, la apertura de la Escuela de Bellas Artes en 1919 significó una luz en ese "opaco" ambiente limeño.

Aunque pocas, por ese entonces otras mujeres también incursionaron en la escritura sobre arte peruano. Entre los nombres que firmaron comentarios artísticos localizamos a las intelectuales Zoila Aurora Cáceres y María Wiesse. El aporte de Cáceres al arte peruano es relevante, pues en la segunda década del siglo XX publicó artículos sobre emblemáticos pintores para la historia del arte peruano, tales como Ignacio Merino y Daniel Hernández. Mientras que Wiesse, luego de dar a conocer algunos artículos comentando exposiciones, incursionó en la crítica de cine. Sin embargo, ninguna de ellas le dedicó más páginas a la actividad artística ejecutada por mujeres en el Perú de la década de 1920 que Elvira García y García ${ }^{10}$.

El quehacer periodístico no fue secundario para ella. Sus artículos confirman el tiempo dedicado a esta labor y la opinión sustentada hace referencia a la seriedad con la que la lleva a cabo. Sobre esto es necesario enfatizar que no solo escribe artículos relacionados con las artistas, sino también toma la pluma para comentar la obra ejecutada por hombres; un ejemplo es la crítica dedicada a la exposición del pintor Bernardo Rivero, en la que destacó la manera "real" de captar la naturaleza en sus obras (García y García, 1927a, p. 3).

Asidua visitante a la Escuela de Bellas Artes y de las escasas salas de exposición limeñas, lo que distingue a García y García de los cronistas culturales de la época es el contacto directo con las artistas. Estrecho vínculo logrado cuando la periodista irrumpe en el mundo íntimo del taller, contacto no frecuente en la época. Articulistas y críticos de arte como Carlos Solari y Luis Varela y Orbegozo solían comentar solo las obras exhibidas en las salas y muy rara vez realizaban entrevistas o notas en las que dejaban traslucir las ideas de los artistas. Pierden, entonces, la oportunidad de ser testigos del proceso creativo y de tener acceso a la obra inacabada o no vista en las salas. 
De esto último trata García y García en el artículo "En el taller de una artista peruana", en el que narró su estancia en el de Rosa Angélica Romero, lo que le permitió constatar "los innumerables cuadros que adornan las habitaciones de la casa" y un álbum de apuntes de impresiones de viajes que le sirve de material para ejecutar sus lienzos (García y García, 1924b, p. 7). Publicado en los primeros días de enero de 1924, este artículo con unas variantes le sirvió de base para la biografía que consignó luego en el libro. Discípula de Rebeca Oquendo, ganadora del concurso Concha de 1902, gracias a García y García se sabe que viajó y llegó a estudiar en una escuela de arte en Francia, allí permaneció dos años para luego volver a Lima y convertirse en uno de esos talentos perdidos ante la falta de oportunidades.

En lo referido a las biografías del capítulo "La mujer en las Bellas Artes", las mismas están elaboradas sobre la base de un esquema que comprende: antecedentes familiares (padres y/o cónyuge), formación artística (en ocasiones especificando el nombre de profesores), características de la obra (género, composición, color, etc.) y, en ciertos casos, las exposiciones en las que participó o los lugares donde se localizan las obras. Como se constatará en los siguientes apartados, los artículos y este capítulo son fuentes valiosas para conocer diversos aspectos de la obra artística de las peruanas antes de 1924.

\section{Enseñanza, lenguaje artístico y custodia de la obra}

La enseñanza artística femenina es uno de los temas recurrentes cuando se trata a las artistas de este periodo. Así, al no contar el Perú con un centro de arte oficial, el aprendizaje había sido restringido al ámbito particular y a la formación autodidacta. Sobre esto, la biografía de Francisca Magan de Caravedo ofrece un panorama antes de la apertura de la Escuela Nacional de Bellas Artes, actividad realizada con frecuencia por las mujeres de élite para quienes el conocimiento de una técnica artística era un pasatiempo, un "adorno":

A su regreso al Perú, ingresó al Colegio de Belén, recibiendo fuera de las lecciones generales que allí se pueden dar, una dirección artística del notable dibujante, doctor Romeo Gago [...].

Acontecimientos de distinto orden, hicieron cambiar de rumbos su 
enseñanza artística, para someterse a la dirección del pintor cubano señor Boudat, y fue entonces, cuando hizo su primera exhibición, con un magnífico óleo de Santa Rosa, que mereció los más francos elogios de la prensa local.

Era necesario que siguiera otra escuela, un tanto más en armonía con la técnica pictórica de la época y la inició en esos secretos, el español Julio de Oñate. (García y García, 1924a, p. 532)

Este puntual interés en señalar la formación recibida por Magan traduce el deseo de dejar establecido que la(s) artista(s) no era(n) improvisada(s), sino que su(s) obra(s) era(n) el fruto de estudio y dedicación. De esta manera, la autora destaca que el aprendizaje era el camino que debía seguir la mujer moderna, pues nutría su inteligencia y perfeccionaba su sensibilidad (García y García, 1928, p. 13).

Aunque valora la guía del maestro, es interesante resaltar que deja notar la importancia de la independencia que debe poseer la labor artística, así lo leemos en la vida de la estudiante de la Escuela de Bellas Artes, Rosa Belón (figura 2) cuando escribió: "Tuvimos oportunidad de apreciar a la artista en su taller, desligada de la dirección del maestro. Es allí donde hace obra propia, donde se desdobla su espíritu" (García y García, 1924b, p. 552).

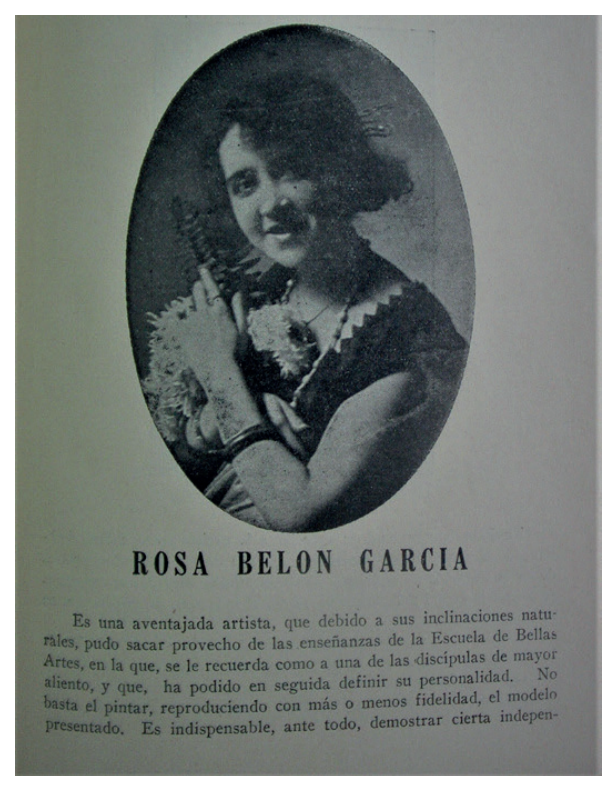

Figura 2. Rosa

Belón García

La biografía de Belón permite también conocer uno de los géneros pictóricos abordados por las primeras profesionales del arte y el impacto del color en la mirada de García y García: 
Es inspirada en todo momento: sus desnudos tienen el valor de la vida que palpita agitando las carnes, cual si sufrieran los efluvios del rubor; en las gasas y telas que se arrugan y entrecruzan suavemente, tomando la más graciosa flexibilidad; en la coloración, siempre fresca, moderada y con toda la belleza de lo que es natural; en la lozanía de las formas; en la seguridad de las posturas en que reposan las figuras, sin dureza ni afección. (García y García, 1924a, p. 553)

Esta vida permite comentar un valor agregado de La mujer peruana a través de los siglos: el de ser, probablemente, el primer libro publicado en el Perú en el que se introducen numerosas imágenes pictóricas de obras ejecutadas por mujeres. Así, solo en este apartado contabilizamos 30 fotografías en las que 17 son obras y las demás los retratos fotográficos de las $\operatorname{artistas~}^{11}$. En tal sentido, la biografía de Belón García tiene cinco fotos, la primera su retrato y las demás de sus obras (figura 3).
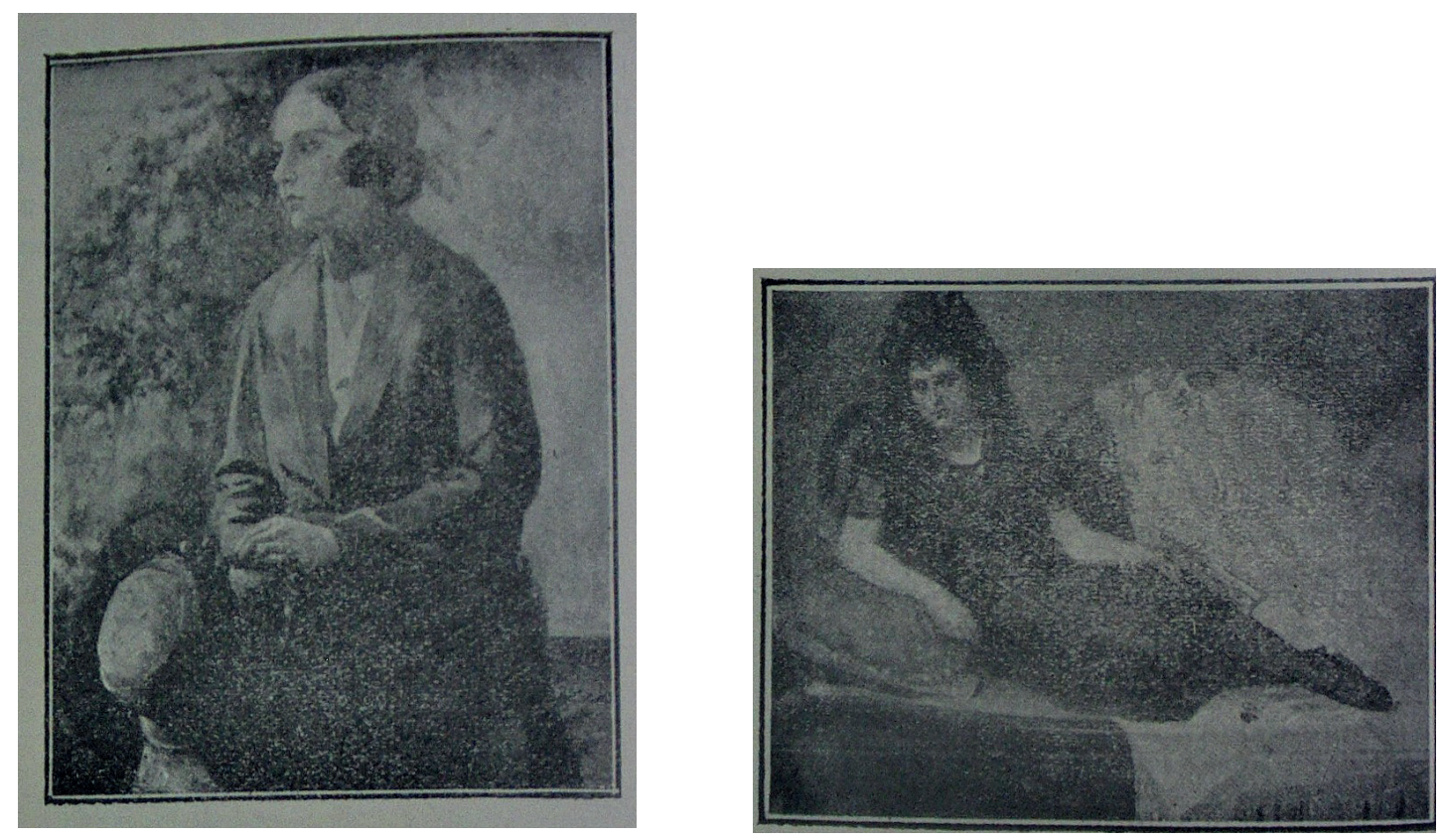

Figura 3. Obras de Rosa Belón: "Meditación” (izquierda) y "Perezosa” (derecha)

Vale la pena especificar que a diferencia del caso del historiador de arte Julio E. Payró, destacado por ser figura clave para el desarrollo de la cultura visual en Argentina, quien "otorgó la misma importancia discursiva tanto a la imagen como a la palabra, generando un relato visual del arte" (Cruz \& Fara, 2013, p. 259), Elvira García y García desaprovechó la imagen de las obras para 
establecer un diálogo entre lo escrito y lo visual. Para ella, la fotografía era un soporte solo referencial que contribuye a destacar las apreciaciones generales que hace a la producción artística.

Al respecto, es también interesante añadir que García y García tuvo una especial admiración por la fotografía, a la que consideraba "obra de arte". Así lo dejó notar en el artículo dedicado a comentar las fotografías de los hermanos arequipeños Vargas, quienes poseían uno de los estudios más famosos de la época y que formaron parte de una generación renovadora de la fotografía en el sur andino peruano. En las últimas líneas, destacó la maestría de los retratos ejecutados por los Vargas en los que valoró "el alma que asoma a los ojos" (García y García, 1932, p. 37). Probablemente, esta idea romántica de la esencia de la personalidad captada en la imagen influyó para que decidiera colocar retratos fotográficos en varias de las biografías del segundo tomo de su obra.

Parte fundamental, complementaria al aprendizaje en aula, era el viaje. Así lo evidencia la autora con su propia experiencia de vida y en algunas biografías, como en la de María Francisca Elguera en la que apuntó que los periplos por Europa le permitieron apreciar la obra de destacados artistas y aprender directamente de ellos. O, en el caso de Isabel Morales Macedo, el viaje le brindó la oportunidad de perfeccionarse cuando, “[d]espués de dos años de estudios en nuestra Escuela de Bellas Artes y cuando había cosechado allí los primeros puestos, resolvió batir las alas y marchar muy lejos", pues, según expresa, "era necesario conocer otros mundos" (García y García, 1924a, p. 562).

Otro asunto tratado en este apartado es lo peruano en la pintura ejecutada en las aulas de la Escuela Nacional de Bellas Artes. Sobre este tema, Elena Izcue captó el interés de la autora, admiración traducida en tres artículos dados a conocer en diversos años. La propuesta de Izcue era distinta a la del indigenismo, que para entonces ya había llamado la atención de varios estudiantes en la Escuela, ella extraía la iconografía del Perú Antiguo para representarla en objetos modernos diversos. A García y García le entusiasmó que ese "arte peruano estilizado" (García y García, 1927b, s/p) pueda aplicarse a telas, lozas y muebles. La escritora 
no se equivoca al comentar con expectativa esta propuesta, pues, años después la artista será reconocida internacionalmente.

Paisajes, desnudos, temas inspirados en lo peruano pero entre los asuntos abordados por las alumnas de la Escuela Nacional de Bellas Artes destaca uno: el retrato y su versión más personalizada, el autorretrato. No es casual que las investigaciones sobre arte pictórico hecho por mujeres en las primeras décadas del siglo XX distingan la práctica de este género como una manera de "existir de forma independiente de la mirada masculina" (Serrano de Haro, 2000, p. 46). Una de las más diestras representantes de este género fue Olga Matellini, cuyos óleos llamaron la atención de García y García por "la postura natural de sus figuras" que armonizan con "el ritmo del movimiento, que se adivina, y con la riqueza del colorido [...] la expresión del rostro es delicado y sugerente" (García y García, 1924a, p. 560).

Otros datos de interés que entregan los escritos de García y García son los referidos a la localización de obras femeninas en museos o en colecciones particulares. En este caso destaca lo que señaló en la biografía de María Teresa Casanave, cuyo párrafo nos ofrece más de una novedad:

La nota mística ha sido predilecta y así pueden contemplarse en los principales templos, los donativos de hermosos cuadros, nacidos de la delicada mano de la señorita Casanave: el retrato del Padre Gual, en los Descalzos; el Descendimiento, en la Recoleta; Cristo en el Monte de los Olivos, en María Auxiliadora (recogido para corregirlo y terminarlo); una hermosa Cabeza de Cristo con la Corona de Espinas, en la iglesia de Tumbes; un Señor de Limpias, al que das últimas pinceladas. Un retrato de gran parecido, del Contralmirante Grau, obsequiado al Ministerio de Marina [...]. (García y García, 1924a, p. 536)

En la actualidad, la obra peruana decimonónica y de los primeros años del siglo XX tiene pocos espacios de exhibición. En el caso específico de la pintura hecha por mujeres es aún más notorio este vacío, pues la mayoría es custodiada por la familia o en colecciones particulares de difícil acceso, lo cual corrobora que lo doméstico no ayuda a conservar la memoria de los sujetos. Por ello, los datos proporcionados por García y García son, en el presente, una fuente invalorable para conocer el destino de varias pinturas creadas por mujeres. 


\section{Abriendo camino}

En febrero de 1929, Elvira García y García publicó el artículo “La mujer peruana y el arte pictórico" en el que señaló: "Quienes hayan visitado la Exposición de trabajos pictóricos, tanto de este año como de los anteriores, tendrán que declarar que la mayor parte de los cuadros expuestos son obra dilecta de la delicada mano femenina" (García y García, 1929a, p. 3). Estas líneas no son halago injustificado; una lectura panorámica de la crítica escrita a partir de las primeras muestras organizadas por la Escuela revela que las obras de las alumnas destacaban en cantidad y, muchas veces, también en calidad.

Lo anterior se constata al conocer lo que Daniel Hernández, primer director de la Escuela Nacional de Bellas Artes, dijo en la memoria de la exposición de alumnos de 1926: "Este año se han distinguido las señoritas alumnas de un modo más notable". Para luego comentar, brevemente, la labor de Elena Izcue, Julia Codesio, Laura Zegarra, María de Rávago y Olga Matellini, las cinco alumnas más destacadas del año, de las cuales — a decir del director — las tres primeras ya habían logrado crear un estilo propio (Escuela Nacional de Bellas Artes, 1926, p. 2). Muestra relevante de que los tímidos talentos habían ya madurado.

No obstante, esta diligente actividad artística femenina, el reconocimiento público del director y el interés de los críticos se contrapone a la respuesta del público limeño. Así lo menciona otro artículo de nuestra autora, en el cual da cuenta de esa apatía:

Cuando un artista se ha lanzado entre nosotros a inaugurar un salón en que exhibir sus obras de arte, siempre hemos pensado que tiene mucho valor, si se consulta la frialdad con que el público de manera general acoge la obra nacional; pero cuando esa decisión es tomada por una mujer, tenemos que admirar mucho más su coraje por lo mismo que no se juzga con justicia y con entera imparcialidad la obra femenina. (García y García, 1929b, p. 3)

El poco entusiasmo por el arte producido en el Perú no era novedad, los grupos con poder adquisitivo preferían comprar la obra de los extranjeros que exhibían en Lima o, en el mejor de los casos, de los peruanos formados o 
radicados en Europa. Desidia que el investigador Mirko Lauer sintetiza en esta línea sobre el mercado del arte limeño antes de 1920: "la protección de las artes ha sido siempre tic individual y no manía colectiva" (1976, p. 47). Dinámica que en algo mejoró con la apertura de la Escuela Nacional de Bellas Artes, por lo menos en lo referido a la difusión de la obra ejecutada por los profesores y estudiantes.

Pero volvamos a la cita de 1929, líneas iniciales de una crítica a la primera individual de la pintora Julia Codesido, cuyo comentario destacó un obstáculo más que debía superar la mujer artista: el prejuicio de género. Tema ampliamente tratado desde la crítica feminista del arte y que conlleva a reflexionar sobre la situación de la mujer artista, luego de haber logrado incursionar en la formación académica, el otro reto fue el de introducir su obra en el mercado del arte.

En tal sentido, la década de 1920 para las alumnas y primeras egresadas de la Escuela Nacional de Bellas Artes es decisiva. Esta generación es la que abre el camino para la configuración de la artista profesional en el Perú. Varias de ellas tomaron otro rumbo, el que la sociedad esperaba, esposas y madres. Pero otras siguieron la ruta de sus anhelos profesionales y lograron imponerse en un campo laboral dominado por los hombres.

Una de las que persistió y triunfó fue, precisamente, la pintora Julia Codesido. Protagonista del artículo citado de García y García, era egresada de la primera promoción de la Escuela Nacional de Bellas Artes y miembro del grupo indigenista. Desde sus primeros años de estudiante, su obra recibió buenos comentarios y augurios. Es decir, calidad plástica no le faltaba, y es lo que el tiempo confirma. Julia Codesido es hoy uno de los principales nombres del arte pictórico peruano de la primera mitad del siglo XX.

\section{Trascendencias}

Una de las maneras de valorar una obra pictórica del pasado es confrontarla con el presente. Encontrar algo en ella con lo cual reflexionar, entender mejor lo que somos o, en el mejor de los casos, plantearnos la posibilidad de modificar nuestra manera de mirar el mundo. Con los libros sucede lo mismo. 
La mujer peruana a través de los siglos y los artículos periodísticos escritos por Elvira García y García a lo largo de la década de 1920 son un caleidoscopio de posibilidades para dar a conocer la obra plástica de las artistas. Hilvanado con un esbozo biográfico, la autora da a conocer la formación artística, viajes, los géneros que practicaban y la localización de la obra.

Estos escritos son más que una recopilación de datos; son testimonios de mujeres, pero también son una muestra de cómo las intelectuales y artistas lograron - con estas alianzas simbólicas - impulsar, difundir y afianzar la labor de sus congéneres en el ámbito social y cultural de las primeras décadas del siglo XX y proyectarlas hasta nuestros días.

\section{Notas}

1 Entre 1896 y 1948, García y García publicó catorce libros. En su tesis, el historiador Edwin Bejarano (2011) divide la temática en tres áreas: libros sobre la mujer, la infancia y la cooperación.

2 Uno de esos momentos es en 1923, cuando a propósito de una exposición industrial, artística y comercial planificada por la revista Mundial escribe un artículo en el que exhorta a los organizadores a destinar una sala a la obra femenina (García y García, 1923, p. 3).

3 En la actualidad, Elvira García y García continúa siendo un referente en la labor educativa. Así, una de las grandes unidades escolares más antiguas de Lima, hoy denominado Colegio Emblemático, lleva su nombre.

4 De aquí en adelante se utilizará este título, forma abreviada y la más conocida para referirse a este libro.

5 Este artículo es un adelanto de mi tesis doctoral dedicada a las primeras profesionales del arte peruano. Agradezco a María Emma Mannarelli su lectura y sugerencias para mejorarlo.

6 Exmilitar, Manuel de Mendiburu es el autor del Diccionario histórico biográfico del Perú (1931-1934). Minucioso trabajo publicado en 1874, corregido y aumentado en la década de 1930 por Evaristo San Cristóval, en cuyas páginas, distribuidas en once volúmenes, incluye la biografía de veinticinco artistas peruanos y extranjeros radicados en el Perú a partir del siglo XVI hasta inicios del XIX. Entre ellos no considera a ninguna artista plástica.

7 En el orden que le otorgó la autora ellas son: Rebeca Oquendo de Subercasseaux, Clotilde Porras de Osma, Rosalía García de Lavalle, Rosa Angélica Romero Sotomayor, María Francisca Elguera, Francisca Magan de Caravedo, María Teresa Casanave, Aurora San Cristóval, Elena Izcue, Julia Codesido, María Rávago Velarde, 
Rosa Belón García, Leonor Vinatea Cantuarias, Olga Mattelini, Eugenia Montagne, Isabel Morales Macedo, Laura Zegarra, Teresa Carvallo, Cristina Malherbe, Rosa Hurwitz y Carmen Saco.

8 Además de su indudable calidad plástica, el principal motivo por el cual Oquendo es la artista peruana más conocida del siglo XIX tiene su explicación en su donativo de cinco lienzos al Estado, algunos de los cuales forman parte de la muestra permanente del Museo de Arqueología, Antropología e Historia del Perú (Pachas Maceda, 2010).

9 Este orden, además de alfabético, corresponde a las investigaciones dedicadas específicamente a la obra de cada una de ellas. El interés por Codesido es de larga data, mientras el de Izcue se dio a partir de la retrospectiva de 1999, cuando empieza a conocerse entre las nuevas generaciones y en el caso de Oquendo son, en su mayoría, breves menciones en libros.

10 Sobre el papel primordial del arte en la obra de Zoila Aurora Cáceres, véase: Pachas, 2009. En tanto, sobre la obra de María Wiesse, cfr.: Wiesse, 2014.

11 Agradezco la colaboración de Jesús Álvarez, quien realizó las tomas fotográficas incluidas en este artículo.

\section{Referencias bibliográficas}

Águila, R. del (2013). (A)filiaciones femeninas: Gorriti y la genealogía de la escritura en Lima. Decimonónicas, 10(1), 45-63. Recuperado de http:// www.decimononica.org/afiliaciones-femeninas-gorriti-y-la-genealogiade-la-escritura-en-lima/

Alvarado Rivera, M. J. (2011). El feminismo. Conferencia leída en la "Sociedad Geográfica” por la señorita María Jesús Alvarado Rivera [1911]. En María Jesús Alvarado Rivera, 1911-2011. Biografia y manifiesto. Lima: Ministerio de la Mujer y Desarrollo Social. Recuperado de https:// www.mimp.gob.pe/files/direcciones/dgignd/campanias/MJAlvarado_ Historia_Manifiesto.pdf

Balta, A. (1998). Presencia de la mujer en el periodismo escrito peruano (18211960). Lima: Universidad de San Martín de Porres.

Bejarano, E. (2011). Elvira García García (1892-1951): Mujer y educadora dentro de los procesos modernizadores de la Educación de la infancia en el Perú (Tesis de licenciatura en Historia). Universidad Nacional Mayor de San Marcos, Lima, Perú. Recuperado de http://cybertesis.unmsm. edu.pe/handle/cybertesis/409

Cáceres, Z. A. (1909). Mujeres de ayer y hoy. París: Garnier. 
Colás Bravo, P. \& Jiménez Cortés, R. (2006). Tipos de conciencia de género del profesorado en el contexto escolar. Revista de Educación, 340, 415-444. Disponible en https://idus.us.es/xmlui/handle/11441/24639.

Contreras, C. \& Cueto, M. (2013). Historia del Perú contemporáneo, 5. edición. Lima: Instituto de Estudios Peruanos.

Cruz, J. y Fara, C. (2013). La difusión del arte a través de la imagen impresa. Julio E. Payró como gestor de lo visual. En L. Malosetti y Gené M. (Comps.), Atrapados por la imagen. Arte y política en la cultura impresa argentina (pp. 255-277). Buenos Aires: Edhasa.

Escuela Nacional de Bellas Artes. (22 de enero de 1926). Memoria correspondiente al año 1925 leída por su director señor Daniel Hernández. El Comercio, p. 2.

García y García, E. (1 de febrero de 1923). Una exposición original. El Comercio, p. 3.

García y García, E. (1924a). La mujer peruana a través de los siglos: serie historiada de estudios y observaciones. Lima: Imprenta Americana. Recuperado de https://ufdc.ufl.edu/AA00019316/00001/3j

García y García, E. (4 de enero de 1924b). En el taller de una artista peruana. El Comercio, p. 7.

García y García, E. (26 de julio de 1926). Una galería de pintura. El Comercio, p. 1.

García y García, E. (22 de febrero de 1927a). Una exposición pictórica. El Comercio, p. 3.

García y García, E. (1927b). El arte peruano. Mundial, 368, s/p.

García y García, E. (1928). Actividad femenina. Lima: Casa Editora "La Opinión Nacional".

García y García, E. (25de febrero de 1929a). La mujer peruana y el arte pictórico. El Comercio, p. 3.

García y García, E. (17 de diciembre 1929b). Una exposición de pinturas. El Comercio, p. 3.

García y García, E. (1 de enero de 1932). El arte fotográfico. El Comercio, p. 37.

García y García, E. (2013). Tendencias de la educación femenina de 1908. En M. Mannarelli. Las mujeres y sus propuestas educativas 1870-1930 (pp. 
225-243). Lima: Fondo Editorial de la Derrama Magisterial.

Guardia, S. (2002). Mujeres peruanas. El otro lado de la historia. Lima: Editorial Minerva.

Gutiérrez de Quintanilla, E. (1924). Prólogo. En E. García y García. La mujer peruana a través de los siglos: serie historiada de estudios y observaciones, tomo I. Lima: Imprenta Americana.

Lauer, M. (1976). Introducción a la pintura peruana del siglo XX. Lima: Mosca Azul Editores.

Mannarelli, M. (2013). Las mujeres y sus propuestas educativas 1870-1930. Lima: Fondo Editorial de la Derrama Magisterial.

Mendiburu, M. (1931-1934). Diccionario histórico biográfico del Perú, 11 vols. Lima: Imprenta Enrique Palacios.

Nochlin, L. (2007). ¿Por qué no han existido grandes artistas mujeres? En K. Cordero \& I. Saenz (Comps.), Crítica feminista en la teoría e historia del arte. Ciudad de México: Universidad Iberoamericana.

Pachas Maceda, S. (2009). Aurora Cáceres “Evangelina”. Sus escritos sobre arte peruano. Lima: Universidad Nacional Mayor de San Marcos.

Pachas Maceda, S. (2010). Rebeca Oquendo. Un legado pictórico por descubrir. Illapa, 7, 11-20. doi: 10.31381/illapa.v0i7.1041

Paz-Soldán, J. (1917). Diccionario biográfico de peruanos contemporáneos. Lima: Librería e Imprenta Gil.

Person, E. (1999). La construcción de la femineidad: su influencia a lo largo del ciclo de vida. En M. Lemlij (Ed.), Mujeres por mujeres (pp. 62-86). Lima: Biblioteca Peruana de Psicoanálisis.

Porras Barrenechea, R. (1954). Fuentes históricas peruanas. Lima: Juan Mejía Baca y P. L. Villanueva Editores.

Serrano de Haro, A. (2000). Modelos de mujer. Mujeres en el arte. Barcelona: Plaza \& Janes Editores.

Wiesse, R. (2014). Letra y música de María Wiesse. Lima: Instituto de Estudios Peruanos. 